\title{
Universal unitary gate for single-photon spinorbit four-dimensional states
}

\author{
Sergei Slussarenko ${ }^{1}$, Ebrahim Karimi ${ }^{1,2}$, Bruno Piccirillo, ${ }^{1,3}$ Lorenzo Marrucci, ${ }^{1,2}$ and Enrico Santamato ${ }^{1,3}$ \\ 1 Dipartimento di Scienze Fisiche, Università di Napoli "Federico II", \\ Compl. Univ. di Monte S. Angelo, 80126 Napoli, Italy \\ 2 CNR-INFM Coherentia, Compl. Univ. di Monte S. Angelo, 80126 Napoli, Italy \\ 3 Consorzio Nazionale Interuniversitario per le Scienze Fisiche della Materia (CNISM), Napoli *
}

\begin{abstract}
The recently demonstrated possibility of entangling opposite values of the orbital angular momentum (OAM) of a photon with its spin enables the realization of nontrivial one-photon spinorbit four-dimensional states for quantum information purposes. Hitherto, however, an optical device able to perform arbitrary unitary transformations on such spinorbit photon states has not been proposed yet. In this work we show how to realize such a "universal unitary gate" device, based only on existing optical technology, and describe its operation. Besides the quantum information field, the proposed device may find applications wherever an efficient and convenient manipulation of the combined OAM and spin of light is required.
\end{abstract}

\section{INTRODUCTION}

Besides energy and linear momentum, photons may carry spin angular momentum (SAM) and orbital angular momentum (OAM). SAM is associated with the polarization and OAM with the transverse amplitude and phase profile of the photon propagation mode $[1,2]$. The photon spin has been one of the most exploited optical resources since the beginning of optics. The photon OAM has received increasing attention in more recent times as a resource in quantum and classical optics, since OAM exists in an inherently multidimensional space. Information can thus be encoded in higher dimensional OAMalphabets $[3,4]$ to be used in free-space communication systems [5] or for increasing the dimensionality of the working Hilbert space in quantum communication systems [6]. In most applications demonstrated thus far, however, only two opposite values of the OAM were allowed, so as to encode one qubit into the OAM of a single photon. A higher (four) dimensional (4D) space can then be still realized by combining the OAM space with the usual spin, or polarization, space, thus realizing what we call a "spinorbit" (SO) space.

In most multidimensional single photon optical schemes realized so far, one qubit has been encoded in the photon spin ("polarization" qubit) and another qubit in the photon linear momentum ("direction" qubit) or even time-bin [7]. The two qubits can be also entangled to each other, thus realizing full four-dimensional states (or ququarts) in the 4D Hilbert space of the photon obtained by the tensor product of the two qubit spaces. This approach requires however to split and recombine the light beam in interferometric configurations which are cumbersome and difficult to maintain aligned. It can be shown that any unitary transformation in the polarization-direction 4D Hilbert space can be realized by using a single interferometer and suitable waveplates,

*Electronic address: enrico.santamato@na.infn.it thus creating an universal unitary gate (UUG) in that space [8]. Replacing the linear momentum with the photon OAM, in quantum information encoding, has the advantage that all processing can be made, in principle, along one beam, without the need for interferometers.

Some manipulations of the photon OAM can be made by using Dove's prisms [9], transverse mode sorters [10], and cylindrical lens mode converters [11], but no UUG has been till now proposed for the photon OAM. However, even if such OAM-UUG were available, its use together with a SAM-UUG would still not reach the goal of a full manipulation of the ququart state encoded in the photon, because these two UUGs would act on the spin and OAM degrees of freedom separately and would not be able to handle two-qubit entangling. Entangled pairs of qubits, i.e., unseparable ququarts, are central in most schemes that have been proposed for quantum communications, quantum information processing and secure key distribution.

In this work, we introduce a UUG acting in the $4 \mathrm{D}$ photon SO space with OAM $m= \pm 2$, where the integer $m$ denotes the value of the photon OAM along the optical beam axis, in units of $\hbar$. Hence, we are restricting the infinite dimensional Hilbert space of the photon OAM to the two-dimensional one $|m|=2$ (all our results are easily generalized to an arbitrary $|m|)$. Our SO-UUG is able to handle arbitrary ququarts in this SO space, including those in which the spin and orbital degrees of freedom of the photon are entangled. Furthermore, our SO-UUG can be adjusted so as to obtain any unitary transformation $U_{s o} \in U(4)$ in the $4 \mathrm{D}$ photon SO-space, that will turn any given input spinorbit state of the photon into any other prescribed output spinorbit state. As a notable application, the spectrum of any physical observable $\hat{A}$ in the SO-space can be measured by means of a suitable SO-UUG adjusted so as to change the eigenvectors of $\hat{A}$ into directly measurable basis states. An important feature of the SO-UUG described here is that this device can be inserted along the beam path without changing its direction and without having recourse to interferometric schemes. Obviously, our SO-UUG is much more complex than the SAM-UUG for photon spin only, and it must 
have 16 adjustable parameters, i.e., as many as the parameters of the $U(4)$ group. Nevertheless, the realization of the SO-UUG is not beyond the possibilities of current technology.

This paper is organized as follows. In the next section we briefly describe the UUG for photon spin only. In Sec. III, we describe the q-plate (QP), a recently invented device that may alter the photon OAM and entangle it with its spin $[12,13,14,15]$. In Sec. IV we introduce the so-called "q-box" (QB), which is the main component of our SO-UUG. The description of the whole SO-UUG is given in Sec. V. In Sec. VI some simple, yet important, examples of ideal SO-UUGs are given. In Sec. VII we discuss the problem of transverse-mode cross-talk in a real SO-UUG, and, finally, in Sec. VIII our conclusions are drawn.

\section{THE SPIN UNITARY GATE}

It is well known that the photon polarization may be easily manipulated by inserting suitable birefringent plates along the beam. A SAM-UUG for the photon spin is realized by an isotropic retardation plate followed by a birefringent half-wave plate (HWP) sandwiched between two birefringent quarter-wave plates (QWP) as shown in the inset of Fig. 1. Changing the retardation $\delta$ of the isotropic plate and the optical axis angles $\alpha, \beta, \gamma$ of the three birefringent waveplates allows one to realize any unitary transformation $\widehat{V}_{s}(\alpha, \beta, \gamma, \delta) \in U(2)$ in the photon polarization space [16]. For example, Pauli's operator, $\hat{\sigma}_{x}, \hat{\sigma}_{y}$, and $\hat{\sigma}_{z}$, in the circular polarization basis of the spin space, may be realized by setting $(\alpha, \beta, \gamma, \delta)=$ $(0, \pi, \pi / 2, \pi / 2) \rightarrow \hat{\sigma}_{x},(\alpha, \beta, \gamma, \delta)=(0, \pi / 4,0, \pi / 2) \rightarrow \hat{\sigma}_{y}$, $(\alpha, \beta, \gamma, \delta)=(0,-\pi / 4, \pi / 2, \pi / 2) \rightarrow \hat{\sigma}_{z}$ (this choice is by no means unique). Denoting as $\left|1_{\hat{S}}, m_{\hat{L}}\right\rangle$ and $\left|-1_{\hat{S}}, m_{\hat{L}}\right\rangle$ the photon states with left and right circular polarization and OAM value $m$ respectively, the action of the SAMUUG is defined by its action on the circular polarization basis states according to

$$
\left(\left|1_{\hat{S}}, m_{\hat{L}}\right\rangle,\left|-1_{\hat{S}}, m_{\hat{L}}\right\rangle\right) \stackrel{\widehat{V}_{s}}{\longrightarrow}\left(\left|1_{\hat{S}}, m_{\hat{L}}\right\rangle,\left|-1_{\hat{S}}, m_{\hat{L}}\right\rangle\right) \mathcal{V}_{s}
$$

where $\mathcal{V}_{s}$ is a $2 \times 2$ unitary matrix.

\section{THE Q-PLATE}

Recently, a new device has been introduced, named q-plate (QP), capable of producing entanglement between the spin and the OAM degrees of freedom of a photon $[12,13,14,15]$. The QP is essentially a retardation wave-plate whose optical axis is aligned nonhomogeneously in the transverse plane in order to create a topological charge $q$ in its orientation. An example of $q$-plate with topological charge $q=1$ is provided by the azimuthal alignement of the local optical axis, as shown in Fig. 1. QPs may be realized using liquid crystals or other suitably patterned birefringent materials. The optical behavior of the QP is characterized by its birefringent retardation $\delta$ and its topological charge $q$. We say the QP to be "tuned" when its optical retardation is $\pi[13,14]$. A tuned QP with charge $q=1$ performs a complete spin-to-OAM conversion of the photon state: left- (right-) circularly polarized photons are converted into right- (left-) handed photons and, simultaneously, the photon OAM value $m$ is changed into $m+2(m-2)$, thus preserving the photon total spinorbit angular momentum $[12,14]$. The tuned QP acts as a polarizationcontrolled OAM mode shifter and can induce entanglement between the spin and OAM degrees of freedom [18]. The action of a tuned QP of charge $q=1$ on the states $\left|1_{\hat{S}}, m_{\hat{L}}\right\rangle$ and $\left|-1_{\hat{S}}, m_{\hat{L}}\right\rangle$ can be summarized as follows

$$
\left(\left|1_{\hat{S}}, m_{\hat{L}}\right\rangle,\left|-1_{\hat{S}}, m_{\hat{L}}\right\rangle\right) \stackrel{\widehat{Q P}}{\rightarrow}\left(\left|-1_{\hat{S}},(m+2)_{\hat{L}}\right\rangle,\left|1_{\hat{S}},(m-2)_{\hat{L}}\right\rangle\right)
$$

In this way, a beam in an OAM state $m= \pm 2$ is transformed into a beam with $m= \pm 4$ or $m=0$, depending on the polarization. The QP, in fact, cannot be considered a gate in our SO-space with $m= \pm 2$, because it brings photons states out of this space. For this reason, any gate acting in SO-space must contain a second cascaded QP which restores the photon into the original OAM subspace.

An important issue that must be taken into account with all photon OAM-shifters as QPs (as well as fork holograms) is the transverse mode non-stationarity under free propagation, due to the beam radial profile arising at the output of these devices. In fact, we should have properly described our photon states by $\left|s_{\hat{S}}, m_{L}\right\rangle\left|\varphi_{m}\right\rangle$, where $\left|\varphi_{m}\right\rangle$ is the radial state, whose evolution during propagation depends on the OAM eigenvalue $m$. The transverse beam profile associated to the radial state $\left|\varphi_{m}\right\rangle$ is given by the radial function $\varphi_{m}(r)=\left\langle r \mid \varphi_{m}\right\rangle$, where $|r\rangle$ denotes the state of a photon localized at radial distance $r$ from the optical axis. In general, the profile $\varphi_{m}(r)$ is a linear combination of infinite Laguerre-Gauss (LG) radial profiles $\mathrm{LG}_{p m}(r)$ with given $m$ and different radial numbers $p$. During free propagation, the Gouy phases of the different LG-mode components change relative to each other, so that the total radial profile $\varphi_{m}(r)$ resulting from the superposition is not stationary. In most quantum computation experiments, where OAM is not involved, the transverse profiles are ignored, since they are the same for every state and can be factorized out. We assumed such a factorization of radial mode in writing Eq. (2), which is justified when the QP is optically thin and the radial mode is already factorized in the input beam. But the right hand side of Eq. 2 shows that the beam emerging from the QP has two different $m$ components, whose initially identical radial profile will change differently during free propagation. As we shall see in a specific example, this will give rise to some transverse "cross-talk" between different $m$-components of the beam reducing the fidelity of our SO-UUG. 


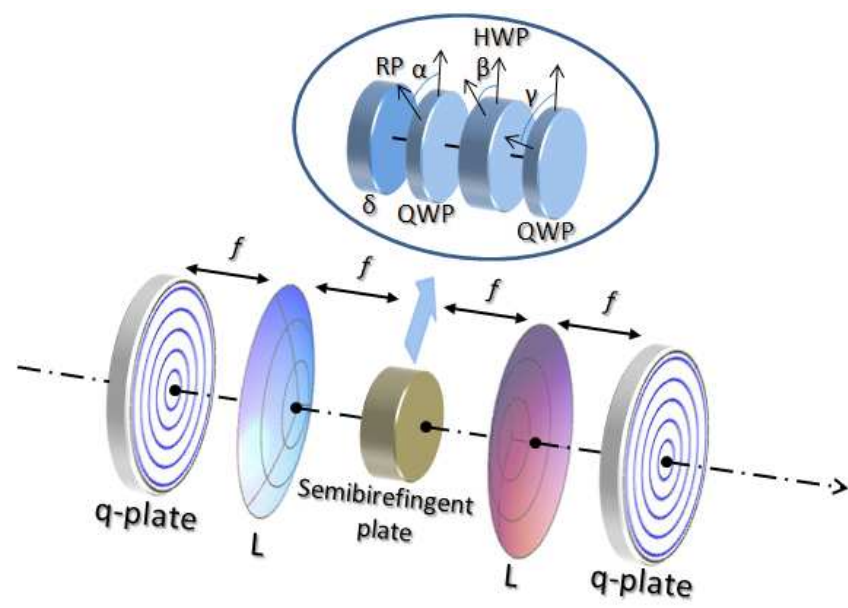

FIG. 1: (Color online) The scheme of the Q-box. The structure of the birefringent plate is shown in the inset. QWP, HWP, RP are the quarter-wave plate, half-wave plate and retardation plate respectively. $\alpha, \beta, \gamma$ indicate rotation of the QWP, HWP, QWP and $\delta$ is the retardation of the isotropic plate. The birefringent plate realizes a SAM-UUG affecting the OAM $m=0$ part of the beam only and it is placed in the common focal plane of the two lenses (L). The local optical axis of both q-plates is tangent to the concentric circles so that $q=1$. The optical retardation both $q$-plate is $\lambda / 2$.

\section{THE Q-BOX}

The basic element of our SO-UUG is the device, here named q-box (QB), that is shown in Fig. 1. The QB is made by cascading two QPs, the first for spliting the OAM $m= \pm 2$ components of the input beam into $m=0$ and $m= \pm 4$ components, and the second to return them back into $m= \pm 2$ components. Between the two QPs, a SAM-UUG is inserted which performs an arbitrary unitary transformation $\widehat{V}_{s}(\alpha, \beta, \gamma, \delta)$ as described in Sec. II on the photon spin only, and which acts selectively on the OAM $m=0$ component of the beam, leaving the $m= \pm 4$ components unaffected. This is made possible, because the $m=0$ and $m= \pm 4$ components of the beam, initially superimposed at the exit plane of the first QP, will spatially separate in their radial pattern by free propagation. In fact, the $m=0$ part will become concentrated in a spot at the beam center, and the $m= \pm 4$ part will become distributed over a surrounding ring with zero intensity at center ("doughnut" beam shape). Having a good separation between the $m=0$ and the $m= \pm 4$ OAM components is crucial for obtaining an efficient SO-UUG. This can be obtained in several ways. In this work we consider a very simple sorting method, based on a semibirefringent circular mask posed at the back focal plane of the first lens shown in Fig. 1. The central part of the mask is a birefringent disk of radius $R$, while the surrounding corona is an isotropic medium. The central disk performs the required unitary transformation $\widehat{V}_{s}$ on the photon spin of the $m=0$ component.
The radius $R$ of the active part of the semibirefringent plate can be adjusted to mimimize the crosstalk among the transverse radial modes. This minimization problem will be discussed in Sec.VII in a specific example. The best spatial separation among radial modes occurs in the far field or, equivalently, at the back focal plane of the first lens in Fig. 1, where the Fourier transform of the field is collected. The second lens in Fig. 1 performs the inverse Fourier transform thus restoring the input beam transverse profile in the final output. To study the behavior of the QB in more detail, it is convenient to use the following logical states in the SO Hilbert space with OAM value $m= \pm 2$ :

$$
\begin{aligned}
& |00\rangle=\left|1_{\hat{S}}, 2_{\hat{L}}\right\rangle, \quad|01\rangle=\left|-1_{\hat{S}},-2_{\hat{L}}\right\rangle \\
& |10\rangle=\left|-1_{\hat{S}}, 2_{\hat{L}}\right\rangle, \quad|11\rangle=\left|1_{\hat{S}},-2_{\hat{L}}\right\rangle .
\end{aligned}
$$

The action of any linear operator $\widehat{V}$ in the spinorbit space is given by its action on the basis states, according to

$$
(|00\rangle,|01\rangle,|10\rangle,|11\rangle) \stackrel{\widehat{V}}{\rightarrow}(|00\rangle,|01\rangle,|10\rangle,|11\rangle) \mathcal{V}
$$

where $\mathcal{V}$ is a $4 \times 4$ matrix. In particular, operators as $\widehat{V}_{s}$ acting only on the photon spin are mapped in spinorbit matrices having the general form

$$
\mathcal{V}_{s}=\left(\begin{array}{cccc}
v_{11} & 0 & v_{12} & 0 \\
0 & v_{22} & 0 & v_{21} \\
v_{21} & 0 & v_{22} & 0 \\
0 & v_{12} & 0 & v_{11}
\end{array}\right)
$$

where $v_{i j}$ are the entries of the $2 \times 2$ matrix $\mathcal{V}_{s} \in U(2)$ in Eq. (1). Similarly, operators $\widehat{U}_{o}$ acting in the OAM subspace only are mapped in spinorbit matrices having the general form

$$
\mathcal{U}_{o}=\left(\begin{array}{cccc}
u_{11} & 0 & 0 & u_{12} \\
0 & u_{22} & u_{21} & 0 \\
0 & u_{12} & u_{11} & 0 \\
u_{21} & 0 & 0 & u_{22}
\end{array}\right)
$$

where $u_{i j}$ are the entries of the $2 \times 2$ matrix $\mathcal{U}_{o} \in U(2)$. Assuming the first QP located in the front focal plane of the first lens of the QB, in the back focal plane we have the 2D-Fourier transform of the field exiting from the QP. The 2D-Fourier transform can be described by an operator $\widehat{J}_{|m|}$ acting only on the radial part of the state: viz. $\widehat{J}_{|m|}\left|s_{\hat{S}}, m_{\hat{L}}, \varphi_{m}\right\rangle=\left|s_{\hat{S}}, m_{\hat{L}}\right\rangle \widehat{J}_{|m|}\left|\varphi_{m}\right\rangle$. In the basis of the localized states $|r\rangle$ in the Fourier plane (lens back focal plane) the matrix elements of the operator $\widehat{J}_{|m|}$ are given by

$$
\left\langle r^{\prime}\left|\widehat{J}_{|m|}\right| r\right\rangle=\frac{1}{\lambda f} J_{|m|}\left(\frac{r^{\prime} r}{\lambda f}\right)
$$

where $J_{|m|}(x)$ are the Bessel functions of integer order $|m|, \lambda$ is the wavelength and $f$ is the lens focal length. The operators $\widehat{J}_{|m|}$ are hermitian and unitary, so that 
$\widehat{J}_{|m|}^{2}=1$. The action of a semi-birefringent plate (SBP) with the birefringent disk of radius $R$ with corresponding unitary spin operator $\widehat{V}_{s}$ is given by

$$
\begin{aligned}
& \left|s_{\hat{S}}, m_{\hat{L}}, \varphi_{m}\right\rangle \stackrel{\widehat{S B P}}{\rightarrow} \\
& \widehat{V}_{s}\left|s_{\hat{S}}, m_{\hat{L}}\right\rangle \widehat{U}(R)\left|\varphi_{m}\right\rangle+\left|s_{\hat{S}}, m_{\hat{L}}\right\rangle(\widehat{I}-\widehat{U}(R))\left|\varphi_{m}\right\rangle(8)
\end{aligned}
$$

Where $\widehat{U}(R)$ is hermitian and idempotent operator that acts only on the radial profile state, selecting the portion of the beam at distance $r \leq R$ and $\widehat{I}$ is the identity operator. In the basis of the localized states $|r\rangle$ the operator $\widehat{U}(R)$ is represented by the Heaviside unit step function $\Theta(R-r)$ with diagonal matrix elements

$$
\left\langle r^{\prime}|\widehat{U}(R)| r\right\rangle=\Theta(R-r) \delta\left(r-r^{\prime}\right)
$$

The action of the QB on the logical basis states (3) can now be easily calculated

$$
\begin{aligned}
& (|00\rangle,|01\rangle,|10\rangle,|11\rangle)\left|\varphi_{2}\right\rangle \stackrel{\widehat{Q}_{b}}{\rightarrow}(|00\rangle,|01\rangle,|10\rangle,|11\rangle) \mathcal{Q}_{b}\left|\varphi_{2}\right\rangle+ \\
& \left(\left(v_{11}-1\right)|01\rangle+\left(v_{22}-1\right)|00\rangle+v_{12}\left|1_{\hat{S}},-6_{\hat{L}}\right\rangle+v_{21}\left|-1_{\hat{S}}, 6_{\hat{L}}\right\rangle\right) \widehat{J}_{4} \widehat{U}(R) \widehat{J}_{4}\left|\varphi_{2}\right\rangle+ \\
& (|10\rangle,|11\rangle)\left(\mathcal{I}-\mathcal{V}_{s}\right) \widehat{J}_{0}(\widehat{I}-\widehat{U}(R)) \widehat{J}_{0}\left|\varphi_{m}\right\rangle
\end{aligned}
$$

where $v_{i j}$ are the entries of the $2 \times 2$ matrix $\mathcal{V}_{s}$ of the birefringent disk, $\mathcal{I}$ is the 2 D-identity matrix and the $\mathcal{Q}_{b}$ is a $4 \times 4$ matrix given by

$$
\mathcal{Q}_{b}\left(\mathcal{V}_{s}\right)=\left(\begin{array}{cccc}
1 & 0 & 0 & 0 \\
0 & 1 & 0 & 0 \\
0 & 0 & v_{11} & v_{12} \\
0 & 0 & v_{21} & v_{22}
\end{array}\right)
$$

The last two terms in the left side of Eq. (10) are the crosstalk terms that appear due to the small overlap of $m=0$ and $m= \pm 4$ modes in the Fourier plane. The effects related to mode cross-talk will be discussed below in a specific example. For the moment, we define the ideal QB a QB where the transverse mode cross-talk is negligible. The action of an ideal QB is then given by

$$
(|00\rangle,|01\rangle,|10\rangle,|11\rangle) \stackrel{\widehat{Q}_{b}}{\rightarrow}(|00\rangle,|01\rangle,|10\rangle,|11\rangle) \mathcal{Q}_{b}
$$

The matrix $\mathcal{Q}_{b}$ in Eq. (11) has not the form of the matrices in Eqs. (5) and (6), showing that the QB acts on both the spin and OAM degree of freedom of the photon simultaneously, producing entangling. Because the matrix $\mathcal{V}_{s}$ of the semi-birefringent plate is unitary, it is evident from Eq. (11) that also $\mathcal{Q}_{b}$ is unitary and so is the action of the ideal QB. The main property of the QB is that although the first QP takes photons out of our initial SO logical space (3), the operator of the full ideal $\mathrm{QB}$ is well defined in this space, as shown by Eq. (12).

\section{THE UNIVERSAL UNITARY GATE}

In this section, we show that the ideal QB defined by Eq. (12) can be used to make the required UUG in our SO space. The QB itself does not provide the most general unitary transformation of $U(4)$. We can see this just by considering that $\mathcal{Q}_{b}\left(\mathcal{V}_{s}\right)$ (as $\mathcal{V}_{s}$ itself) depends on four real parameters only, while the most general unitary operator $U_{s o} \in U(4)$ has 16 free parameters. The UUG in the spinorbit space is therefore a device more complex than the QB. It is then remarkable that the sequence of

- an ideal QB $\mathcal{Q}_{b}\left(\mathcal{V}_{2}\right)$

- a quarter-wave plate at angle 0

- an ideal $\mathrm{QB} \mathcal{Q}_{b}\left(\mathcal{V}_{R}\right)$

- a half-wave plate at angle 0

- an ideal $\mathrm{QB} \mathcal{Q}_{b}\left(\mathcal{V}_{L}\right)$

- a quarter-wave plate at angle 0

- an ideal $\mathrm{QB} \mathcal{Q}_{b}\left(\mathcal{V}_{1}\right)$

provides the required UUG in the SO Hilbert space. Here $\mathcal{V}_{1}, \mathcal{V}_{R}, \mathcal{V}_{L}, \mathcal{V}_{2}$ are the $2 \times 2$ unitary matrices characterizing the semi-birefringent plates inserted in each QB. We notice that all elements of the QB are transparent so that many of them can be cascaded along the beam direction maintaining optical losses at reasonable level. The SO-UUG described above has the proper number of free parameters and it is unitary. However, we should also demonstrate that it is universal, i.e. that any unitary matrix of $\mathcal{U}_{s o} \in U(4)$ can be realized by the sequence above. The proof is based on the results by Englert et al. [8]. In fact, a straightforward calculation shows that the matrix $\mathcal{U}_{\text {so }}$ associated to our UUG has the block form

$$
\mathcal{U}_{s o}=\left(\begin{array}{ll}
\mathcal{S}_{L L} & \mathcal{S}_{L R} \\
\mathcal{S}_{R L} & \mathcal{S}_{R R}
\end{array}\right)
$$


where the $2 \times 2$ blocks are given by

$$
\begin{aligned}
\mathcal{S}_{R R} & =\frac{1}{2} \mathcal{V}_{2}\left(\mathcal{V}_{R}+\mathcal{V}_{L}\right) \mathcal{V}_{1} \\
\mathcal{S}_{L L} & =\frac{1}{2}\left(\mathcal{V}_{R}+\mathcal{V}_{L}\right) \\
\mathcal{S}_{R L} & =-\frac{i}{2} \mathcal{V}_{2}\left(\mathcal{V}_{R}-\mathcal{V}_{L}\right) \\
\mathcal{S}_{L R} & =\frac{i}{2}\left(\mathcal{V}_{R}-\mathcal{V}_{L}\right) \mathcal{V}_{1}
\end{aligned}
$$

Equations (13) and (14) are identical to Eqs. (17) and (18) of Ref. [8], where it is also shown that for any given unitary matrix $\mathcal{U}_{s o} \in U(4)$ one can find four unitary matrices $\mathcal{V}_{1}, \mathcal{V}_{R}, \mathcal{V}_{L}, \mathcal{V}_{2} \in U(2)$ such that $\mathcal{U}_{\text {so }}$ has the form (13) with (14). It is worth noting that all manipulations in our SO-UUG are made on the photon spin degree of freedom, and that QPs are used to transfer the required operations to the OAM degree of freedom, as in Ref. [15].

One SO-UUG can perform any $U(4)$ transformation in the SO space with $m= \pm 2$. However, many nontrivial operations can be realized with simpler devices. For example, a straightforward calculation shows that one QB with a half-wave plate inside is enough to implement the c-NOT operator on our logical state $(|00\rangle \rightarrow|00\rangle,|01\rangle \rightarrow$ $|01\rangle,|10\rangle \rightarrow|11\rangle,|11\rangle \rightarrow|10\rangle)$. A more common set of logical states in the photon spinorbit space is the set where the first bit corresponds to the photon spin and the second bit to the photon OAM:

$$
\begin{aligned}
& |0,0\rangle=\left|1_{\hat{S}}, 2_{\hat{L}}\right\rangle, \quad|0,1\rangle=\left|1_{\hat{S}},-2_{\hat{L}}\right\rangle \\
& |1,0\rangle=\left|-1_{\hat{S}}, 2_{\hat{L}}\right\rangle, \quad|1,1\rangle=\left|-1_{\hat{S}},-2_{\hat{L}}\right\rangle .
\end{aligned}
$$

In this "natural" set of basis states, the action of our SOUUG is different. For example, the matrix $\mathcal{Q}_{n}$ associated to a single QB in the "natural" states basis assumes the form

$$
\mathcal{Q}_{n}\left(\mathcal{V}_{s}\right)=\left(\begin{array}{cccc}
1 & 0 & 0 & 0 \\
0 & v_{11} & v_{12} & 0 \\
0 & v_{21} & v_{22} & 0 \\
0 & 0 & 0 & 1
\end{array}\right)
$$

in place of Eq. (11). This way, the single QB which realizes the c-NOT operation in the logical states basis (3), on the states (15) performs the swapping operation $(|00\rangle \rightarrow|00\rangle,|01\rangle \rightarrow|10\rangle,|10\rangle \rightarrow|01\rangle,|11\rangle \rightarrow|11\rangle)$. Since the choice (15) of states is the most used in the literature, we will use this "natural" basis hereafter. Among the gates that can be realized by the UUG, some are more important than others. Here we list some of these useful gates indicating how they can be implemented. It is worth noting, however, that the implementation of a gate is not unique, and the gates presented here can be realized, in some cases, in simpler ways.

\section{EXAMPLES}

All examples shown in this section have been worked out in the "natural" basis of logical states (15). We have already seen, that the swapping gate is made of one QB with a HWP (i.e. $\mathcal{V}_{s}=\sigma_{x}$ ) semibirefringent plate. The swapping gate is very useful, because it allows one to transfer any unitary action made on the spin qubit to the OAM qubit. For example, a gate mapping basis states into equal-weight maximally entangled orthogonal superpositions (called Hadamard gate), for the single-qubit in the spin degree of freedom, is simply realized by a QWP oriented at $45^{\circ}$. Insertion of the swapping gate after the QWP yields a Hadamard gate acting onto the OAM qubit, leaving the spin qubit unchanged for future manipulation. A Hadamard gate for general ququarts can be realized with three QBs by setting $\mathcal{V}_{1}=\sigma_{y}, \mathcal{V}_{2}=i \sigma_{z}, \mathcal{V}_{L}=\sigma_{x}, \mathcal{V}_{R}=1$

Another useful gate is the c-NOT gate. The c-NOT gate, realized by UUG, can be either a spin-controlled or a OAM-controlled NOT gate. The difference between them is whether the control bit is encoded in the SAM qubit or in the OAM qubit, respectively. These two gates are given by Eqs. (13) and (14) with $\mathcal{V}_{1}=$ $-i \sigma_{x}, \mathcal{V}_{2}=i \sigma_{x}, \mathcal{V}_{L}=\sigma_{z}, \mathcal{V}_{R}=1$ for the spin c-NOT and $\mathcal{V}_{1}=-i, \mathcal{V}_{2}=i, \mathcal{V}_{L}=\sigma_{z}, \mathcal{V}_{R}=1$ for the OAM c-NOT, respectively. These gates are very useful in most quantum optics applications because of their universality.

A measurement of Bell states can be performed with a gate, which transforms Bell state basis in the natural one, where each state can be separated by common devices. The Bell's state measurement gate is provided by $\mathcal{V}_{1}=1, \mathcal{V}_{2}=i \sigma_{y}, \mathcal{V}_{L}=\mathcal{V}_{R}=1 / \sqrt{2}\left(1-i \sigma_{y}\right)$. This gate unties the entanglement between the photon SAM and OAM allowing to measure the two degrees of freedom separately.

\section{THE RADIAL MODES CROSSTALK}

A final issue to be addressed to is the "crosstalk" among radial modes taking place in our device. This effect lowers, in general, the overall quality of the QB (and hence of the SO-UUG) and must be maintained at tolerable levels. As it can be seen from Eq. (10), when some overlap of $m=0$ and $m= \pm 4$ modes is present, crosstalk terms appear in the QB.

We made an evaluation of the effects due to the radial cross-talk in the case of the swapping gate (one QB with half-wave semibirefringent plate), using the radial Laguerre-Gauss mode $\mathrm{LG}_{02}$ profile as input [17]. The radial field profiles of the $m=0$ and $m= \pm 4$ OAM components of the beam in the back focal plane of the first lens are shown in Fig. 2. We notice that, although the main part of the power of the $m=0$ component is concentrated at the beam center, where the $m= \pm 4$ component vanishes, a small crosstalk is present due to the Airy secondary maxima of the $m=0$ mode. As indication of the quality of the QB as an element of the unitary gate we used the "fidelity" of the photon state $\left|\psi_{\text {real }}\right\rangle$ produced by the swapping QB with respect to the state $\left|\psi_{\text {ideal }}\right\rangle$ that an ideal QB would have produced. The 
fidelity $F$ is here defined by $F=\left|\left\langle\psi_{\text {real }} \mid \psi_{\text {ideal }}\right\rangle\right|$. The input photon state and the expected state from an ideal swapping gate are given by

$$
\left|\psi_{\text {in }}\right\rangle=(a|0,0\rangle+b|0,1\rangle+c|1,0\rangle+d|1,1\rangle)\left|\mathrm{L} G_{02}\right\rangle
$$

and

$$
\left|\psi_{\text {ideal }}\right\rangle=(a|0,0\rangle+c|0,1\rangle+b|1,0\rangle+d|1,1\rangle)\left|\mathrm{L}_{02}\right\rangle
$$

respectively. The ideal QB given by Eq. (16) with $\mathcal{V}_{s}=$ $\sigma_{x}$ has $100 \%$ fidelity with respect to the output state $\psi_{\text {ideal }}$. However, the action of a real swapping gate on the input state (17) is described by

$$
\begin{aligned}
& (a|0,0\rangle+b|0,1\rangle+c|1,0\rangle+d|1,1\rangle)\left|\mathrm{L} G_{02}\right\rangle \stackrel{\text { Swap }}{\rightarrow} \\
& (a|0,0\rangle+c|0,1\rangle+b|1,0\rangle+d|1,1\rangle)\left|\mathrm{L} G_{02}\right\rangle- \\
& {\left[(c-b)(|1,0\rangle+|0,1\rangle) \widehat{J}_{0}(\widehat{I}-\widehat{U}(R)) \widehat{J}_{0}-\right.} \\
& \left(a\left(|0,0\rangle-\left|-1_{\hat{S}}, 6_{\hat{L}}\right\rangle\right)+\right. \\
& \left.\left.d\left(|1,1\rangle-\left|1_{\hat{S}},-6_{\hat{L}}\right\rangle\right)\right) \widehat{J}_{4} \widehat{U}(R) \widehat{J}_{4}\right]\left|\mathrm{L} G_{02}\right\rangle
\end{aligned}
$$

Assuming $b$ and $c$ to be real-valued, we can pose $\left(x^{2}=\right.$ $\left.1-a^{2}-d^{2}\right)$

$$
b=x \cos \theta, \quad c=x \sin \theta .
$$

The swapping gate fidelity function is then given by

$$
F=\left|1-x^{2}(1-\sin 2 \theta)\left(1-\gamma_{0}(R)\right)-\left(1-x^{2}\right) \gamma_{4}(R)\right|(21)
$$

where $\gamma_{m}(R)=\left\langle\mathrm{L} G_{02}\left|\widehat{J}_{m} \widehat{U}(R) \widehat{J}_{m}\right| \mathrm{L} G_{02}\right\rangle$ account for the radial mode cross-talk. The parameters $\gamma_{m}(R)$ depend on the radius $R$ of the active disk of the SBP inserted in the QB. In the case of the ideal QB, we have $\gamma_{0}(R) \rightarrow 1$, $\gamma_{4}(R) \rightarrow 0$, and $F \rightarrow 1$. We notice that the fidelity $F$ given by Eq. (21) depends on the input state of the photon. The knowledge of the state to be swapped allows us to select the radius $R$ of the birefringent disk so as to have maximum fidelity (that can vary from $83 \%$ to almost $100 \%$, in some cases). A good compromise, however, can be obtained choosing $R=2.928$ (in units of beam waist at the back focal plane), which gives us a flat minimum fidelity of $83.7 \%$ for any input state. Among the other ways to increase the fidelity of QB, one could, for example, put a non-transparent belt around the birefringent disk in order to absorb the photons in the modeoverlapping region. This method can increase the fidelity of the QB up to $100 \%$ at the price of decreasing transmission efficiency of the device. Another method could be to adjust the input beam profile or improving the design of the $q$-plate so as to minimize crosstalk of $m=0$ and $m= \pm 4$ modes. The use of higher-order OAM modes could also help. We finally note that the full spinorbit UUG has many reflecting surfaces (about 70) so that high quality multireflection coating of all surfaces is highly desirable[19].

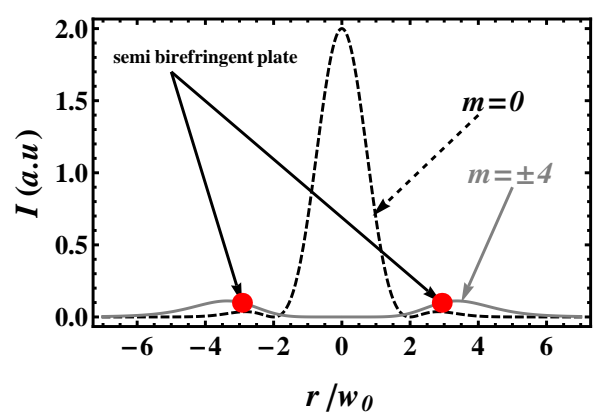

FIG. 2: (Color online) The transverse intensity profiles produced by an incident $\mathrm{LG}_{0,2}$ beam at the back focal plane of the first lens in Fig. 1, where the semi-birefringent plate is inserted. The two curves have been normalized to unit area, for better showing the mode crosstalk.

\section{CONCLUSIONS}

In conclusion, we have introduced a new optical device, the $\mathrm{QB}$, by which universal unitary gates can be assembled to realize arbitrary unitary transformations $U_{s o} \in U(4)$ in the photon spinorbit Hilbert space. All optical manipulations are made by SAM-UUG acting on the photon spin alone, and their action is transferred to the OAM degree of freedom by means of QPs. This introduces a practical advantage, because the photon spin can be easily and rapidly manipulated by using birefringent materials. Our SO-UUG is highly transparent, which guarantees a high photon transmission efficiency, and can be inserted along the beam path in as much the same way as other optical components are inserted without changing the beam direction. This allows us to perform nontrivial two-qubit handling with optical assemblies having much less overall size and much more stability against environmental noise than interferometers. We think that QB and QB-based UUG could have an impact in all fields where complete manipulation of the light OAM and polarization is needed such as, for instance, in optical tweezers and traps, optical communications, optical computing and fundamental quantum optics.
[1] L. Allen, M. W. Beijersbergen, R. J. C. Spreeuw, and J. P. Woerdman, Phys. Rev. A 45, 8185 (1992).

[2] S. Franke-Arnold, L. Allen, and M. Padgett, Laser Photonics Rev. 2, 299 (2008).
[3] G. Molina-Terriza, J. P. Torres, and L. Torner, Phys. Rev. Lett. 88, 013601 (2002).

[4] G. Molina-Terriza, J. P. Torres, and L. Torner, Nat. Phys. 3, 305 (2007). 
[5] G. Gibson et al., Opt. Express 12, 5448 (2004).

[6] A. Mair, A. Vaziri, G. Welhs, and A. Zeilinger, Nature 412, 313 (2001).

[7] I. Marcikic et al., Phys. Rev. A 66, 062308 (2002).

[8] B.-G. Englert, C. Kurtsiefer, and H. Weinfurter, Phys. Rev. A 63, 032303;1 (2001).

[9] N. González, G. Molina-Terriza, and J. P. Torres, Opt. Express 14, 9093 (2006).

[10] H. Sasada and M. Okamoto, Phys. Rev. A 68, 012323 (2003).

[11] M. W. Beijersbergen, L. Allen, H. van der Veen, and J. P. Woerdman, Opt. Commun. 96, 123 (1993).

[12] L. Marrucci, C. Manzo, and D. Paparo, Phys. Rev. Lett. 96, 163905 (2006).

[13] E. Karimi, B. Piccirillo, E. Nagali, L. Marrucci, and E. Santamato, Appl. Phys. Lett. 94, 231124 (2009).
[14] E. Karimi, B. Piccirillo, L. Marrucci, and E. Santamato, Opt. Lett. 34, 1225 (2009).

[15] E. Nagali et al., Phys. Rev. Lett. 103, 013601 (2009).

[16] W. Swindell, editor, Polarized Light, Benchamrk Papers in Optics/1, Dowden, Hutchinson, and Ross, Stroudsburg, 1975.

[17] E. Karimi, G. Zito, B. Piccirillo, L. Marrucci, and E. Santamato, Opt. Lett. 32, 1 (2007).

[18] Such entanglement cannot be achieved with polarizationindependent mode shifters such as fork holograms and spatial light modulators, for example.

[19] With $1 \%$ reflection at each surface the total transmittance of the UUG is reduced to about $50 \%$. With $0.1 \%$ reflection, the transmittance can be still larger than $90 \%$. 